\title{
Spin-dependent localized Hartree-Fock density-functional approach for the accurate treatment of inner-shell excitation of closed-shell atoms
}

\author{
Zhongyuan Zhou ${ }^{1,2}$ and Shih-I Chu ${ }^{1}$ \\ ${ }^{1}$ Department of Chemistry, University of Kansas, Lawrence, Kansas 66045, USA \\ ${ }^{2}$ Department of Physics and Astronomy, University of Kansas, Lawrence, Kansas 66045, USA \\ (Received 19 June 2006; revised manuscript received 19 September 2006; published 17 January 2007)

\begin{abstract}
A spin-dependent localized Hartree-Fock density-functional approach is presented for the efficient and accurate treatment of inner-shell excited states of atomic systems. The approach is applied to the calculation of the total and excitation energies of inner-shell excited states of several closed-shell atomic systems: $\mathrm{Be}, \mathrm{B}^{+}, \mathrm{Ne}$, and $\mathrm{Mg}$. The predicted results are in overall good agreement with available experimental and other $a b$ initio theoretical data. In addition, results for highly excited inner-shell states are presented.
\end{abstract}

DOI: 10.1103/PhysRevA.75.014501

PACS number(s): 31.15.Ew, 32.80.Wr, 32.80.Rm

Recently there is considerable interest in the experimental study of the inner-shell photoionization of atoms and molecules $[1,2]$. The dynamics of inner-shell photoionization and the resulting relaxation of the excited atom are complicated by the collective response of all the electrons of the atomic target. Furthermore, the correlated response of the electrons in the atom couples the photoionization and relaxation processes. Thus, theoretical study of the inner-shell excited

TABLE I. Total energies $(E)$ and excitation energies $(\Delta E)$ of inner-shell excited states $1 s^{-1} n p^{1,3} P(n=2 \sim 8)$ of Be. The groundstate energy is -14.6575 (a.u.).

\begin{tabular}{|c|c|c|c|c|c|}
\hline & $-E$ (a.u.) & & $\Delta E(\mathrm{eV})$ & & \\
\hline States & $\begin{array}{l}\text { Present } \\
\text { work }^{\mathrm{a}}\end{array}$ & $\begin{array}{l}\text { Other } \\
\text { theory }\end{array}$ & $\begin{array}{l}\text { Present } \\
\text { work }^{\mathrm{a}}\end{array}$ & $\begin{array}{l}\text { Other } \\
\text { theory }\end{array}$ & Expt. \\
\hline \multirow[t]{2}{*}{$1 s^{-1} 2 p^{3} P$} & \multirow[t]{2}{*}{10.4526} & $10.4628^{b}$ & \multirow[t]{2}{*}{114.4221} & \multirow[t]{2}{*}{$114.4304^{\mathrm{b}}$} & \\
\hline & & $10.4654^{\mathrm{c}}$ & & & \\
\hline \multirow[t]{2}{*}{${ }^{1} P$} & \multirow[t]{2}{*}{10.4117} & $10.4146^{\mathrm{b}}$ & 115.5350 & $115.7420^{\mathrm{b}}$ & $115.49^{\mathrm{d}}$ \\
\hline & & $10.4209^{\mathrm{c}}$ & & $115.513^{\mathrm{c}}$ & \\
\hline \multirow{3}{*}{$\begin{array}{r}1 s^{-1} 3 p{ }^{3} P \\
{ }^{1} P\end{array}$} & \multirow{3}{*}{$\begin{array}{l}10.1843 \\
10.1797\end{array}$} & $10.1942^{\mathrm{b}}$ & 121.7229 & $121.7395^{\mathrm{b}}$ & \\
\hline & & $10.1882^{\mathrm{b}}$ & 121.8481 & $121.9028^{\mathrm{b}}$ & $121.42^{\mathrm{d}}$ \\
\hline & & $10.2073^{\mathrm{c}}$ & & $121.420^{\mathrm{c}}$ & $121.4^{\mathrm{e}}$ \\
\hline \multirow[t]{3}{*}{$1 s^{-1} 4 p^{3} P$} & \multirow{3}{*}{$\begin{array}{l}10.1410 \\
10.1392\end{array}$} & $10.1504^{b}$ & 122.9012 & $122.9314^{\mathrm{b}}$ & \\
\hline & & $10.1480^{b}$ & 122.9502 & $122.9967^{\mathrm{b}}$ & $122.52^{\mathrm{d}}$ \\
\hline & & $10.1662^{\mathrm{c}}$ & & $122.537^{\mathrm{c}}$ & $122.5^{\mathrm{e}}$ \\
\hline \multirow[t]{3}{*}{$1 s^{-1} 5 p^{3} P$} & \multirow{3}{*}{$\begin{array}{l}10.1239 \\
10.1229\end{array}$} & $10.1331^{\mathrm{b}}$ & 123.3665 & $123.4021^{\mathrm{b}}$ & \\
\hline & & $10.1319^{b}$ & 123.3937 & $123.4348^{\mathrm{b}}$ & $122.96^{\mathrm{d}}$ \\
\hline & & $10.1495^{\mathrm{c}}$ & & $122.992^{c}$ & $123.0^{\mathrm{e}}$ \\
\hline \multirow[t]{2}{*}{$1 s^{-1} 6 p^{3} P$} & \multicolumn{2}{|l|}{10.1152} & 123.6033 & & \\
\hline & \multicolumn{2}{|c|}{$10.114610 .1412^{c}$} & 123.6196 & $123.219^{c}$ & $123.16^{\mathrm{d}}$ \\
\hline \multirow[t]{2}{*}{$1 s^{-1} 7 p^{3} P$} & \multicolumn{2}{|c|}{10.1102} & 123.7393 & & \\
\hline & \multicolumn{2}{|l|}{10.1098} & 123.7502 & & \\
\hline \multirow{2}{*}{$\begin{array}{r}1 s^{-1} 8 p{ }^{3} P \\
{ }^{1} P\end{array}$} & \multicolumn{2}{|l|}{10.1070} & 123.8264 & & \\
\hline & \multicolumn{2}{|l|}{10.1068} & 123.8318 & & \\
\hline
\end{tabular}

${ }^{\mathrm{a}}$ From PW potential.

${ }^{\mathrm{b}} \mathrm{WF}[14]$.

${ }^{\mathrm{c}} \mathrm{SPCR}[15,16]$.

${ }^{\mathrm{d}}$ References [17,18].

${ }^{\mathrm{e}}$ Reference [19]. states, taking into account both exchange and correlation effects accurately, is a subject of current interest. In this paper we focus on the study of the electron structure of inner-shell excited states of closed-shell atoms by means of a spindependent localized Hartree-Fock (SLHF) density-functional approach [3] recently developed, along with the generalized gradient approximation (GGA) treatment of the electron correlation.

The SLHF approach is based on an extension of the exchange (X)-only localized Hartree-Fock (LHF) densityfunctional theory (DFT) [4] to the excited states. In the original X-only LHF DFT [4], developed and successfully used for the treatment of the ground-state calculations of atomic and molecular systems, the basic equation is the Kohn-Sham (KS) equation and the exchange potential is a LHF exchange potential derived under the assumption that the X-only KS

TABLE II. Total energies $(E)$ and excitation energies $(\Delta E)$ of inner-shell excited states $1 s^{-1} n p^{1,3} P(n=2 \sim 8)$ of $\mathrm{B}^{+}$. The groundstate energy is -24.3284 (a.u.).

\begin{tabular}{|c|c|c|c|c|}
\hline States & $\begin{array}{c}-E \text { (a.u.) } \\
\text { Present } \\
\text { work }^{\mathrm{a}}\end{array}$ & $\begin{array}{c}\Delta E(\mathrm{eV}) \\
\text { Present } \\
\text { work }^{\mathrm{a}}\end{array}$ & $\begin{array}{l}\text { Other } \\
\text { theory }\end{array}$ & Expt. $^{\mathrm{c}}$ \\
\hline $1 s^{-1} 2 p^{3} P$ & 17.2559 & 192.4546 & 192.460 & \\
\hline${ }^{1} P$ & 17.1837 & 194.4187 & 194.394 & 194.39 \\
\hline $1 s^{-1} 3 p^{3} P$ & 16.5968 & 210.3878 & 209.850 & \\
\hline${ }^{1} P$ & 16.5861 & 210.6797 & 210.125 & 210.14 \\
\hline $1 s^{-1} 4 p^{3} P$ & 16.4550 & 214.2478 & 213.611 & \\
\hline${ }^{1} P$ & 16.4509 & 214.3599 & 213.715 & 213.76 \\
\hline $1 s^{-1} 5 p^{3} P$ & 16.3959 & 215.8549 & 215.189 & \\
\hline${ }^{1} P$ & 16.3939 & 215.9099 & 215.237 & 215.30 \\
\hline $1 s^{-1} 6 p^{3} P$ & 16.3654 & 216.6860 & 215.999 & \\
\hline${ }^{1} P$ & 16.3642 & 216.7170 & 216.028 & 216.10 \\
\hline $1 s^{-1} 7 p^{3} P$ & 16.3477 & 217.1684 & & \\
\hline${ }^{1} P$ & 16.3469 & 217.1886 & & \\
\hline $1 s^{-1} 8 p^{3} P$ & 16.3362 & 217.4803 & & \\
\hline${ }^{1} P$ & 16.3359 & 217.4895 & & \\
\hline
\end{tabular}

${ }^{\mathrm{a}}$ Form PW potential.

${ }^{\mathrm{b}} \mathrm{DF}$ results [20].

${ }^{\mathrm{c}}$ Reference [20]. 
TABLE III. Total energies $(E)$ and excitation energies $(\Delta E)$ of inner-shell excited states $1 s^{-1} n s^{1,3} S$ and $1 s^{-1} n p^{1,3} P(n=3 \sim 8)$ of Ne. The ground-state energy is -128.9331 (a.u.).

\begin{tabular}{|c|c|c|c|c|c|}
\hline States & $\begin{array}{c}-E \text { (a.u.) } \\
\text { Present } \\
\text { work }^{\mathrm{a}}\end{array}$ & $\begin{array}{l}\text { Other } \\
\text { theory }^{b}\end{array}$ & $\begin{array}{c}\Delta E(\mathrm{eV}) \\
\text { Present } \\
\text { work }^{\mathrm{a}}\end{array}$ & $\begin{array}{l}\text { Other } \\
\text { theory }\end{array}$ & Expt. \\
\hline $1 s^{-1} 3 s^{3} S$ & 97.1495 & 97.1729 & 864.8826 & $864.3917^{\mathrm{b}}$ & \\
\hline${ }^{1} S$ & 97.1411 & 97.1631 & 865.1112 & $\begin{array}{c}864.6583^{b} \\
865.37^{c}\end{array}$ & $\begin{array}{c}865.1^{\mathrm{d}} \\
865.32^{\mathrm{e}}\end{array}$ \\
\hline $1 s^{-1} 4 s^{3} S$ & 97.0348 & & 868.0038 & & \\
\hline${ }^{1} S$ & 97.0326 & & 868.0636 & $868.21^{\mathrm{c}}$ & \\
\hline $1 s^{-1} 5 s^{3} S$ & 97.0008 & & 868.9290 & & \\
\hline${ }^{1} S$ & 97.0000 & & 868.9507 & $869.06^{\mathrm{c}}$ & \\
\hline $1 s^{-1} 6 s^{3} S$ & 96.9863 & & 869.3235 & & \\
\hline${ }^{1} S$ & 96.9859 & & 869.3344 & $869.44^{c}$ & \\
\hline $1 s^{-1} 7 s^{3} S$ & 96.9788 & & 869.5276 & & \\
\hline${ }^{1} S$ & 96.9786 & & 869.5331 & & \\
\hline $1 s^{-1} 8 s^{3} S$ & 96.9744 & & 869.6474 & & \\
\hline${ }^{1} S$ & 96.9743 & & 869.6501 & & \\
\hline $1 s^{-1} 3 p^{3} P$ & 97.0766 & 97.0982 & 866.8663 & $866.4244^{\mathrm{b}}$ & \\
\hline${ }^{1} P$ & 97.0736 & 97.0950 & 866.9480 & $\begin{array}{c}866.5115^{\mathrm{b}} \\
867.18^{\mathrm{c}}\end{array}$ & $\begin{array}{l}867.05^{\mathrm{d}} \\
867.12^{\mathrm{e}}\end{array}$ \\
\hline $1 s^{-1} 4 p^{3} P$ & 97.0151 & 97.0330 & 868.5398 & $868.1986^{\mathrm{b}}$ & \\
\hline${ }^{1} P$ & 97.0141 & 97.0318 & 868.5671 & $\begin{array}{c}868.2312^{b} \\
868.70^{c}\end{array}$ & $\begin{array}{l}868.68^{\mathrm{d}} \\
868.69^{\mathrm{e}}\end{array}$ \\
\hline $1 s^{-1} 5 p^{3} P$ & 96.9919 & 97.0098 & 869.1712 & $868.8299^{b}$ & \\
\hline${ }^{1} P$ & 96.9915 & 97.0095 & 869.1820 & $\begin{array}{c}868.8462^{\mathrm{b}} \\
869.32^{\mathrm{c}}\end{array}$ & $\begin{array}{l}869.23^{\mathrm{d}} \\
869.27^{\mathrm{e}}\end{array}$ \\
\hline $1 s^{-1} 6 p^{3} P$ & 96.9814 & 96.9900 & 869.4569 & $869.1238^{\mathrm{b}}$ & \\
\hline${ }^{1} P$ & 96.9812 & 96.9988 & 869.4623 & $\begin{array}{c}869.1347^{\mathrm{b}} \\
869.58^{\mathrm{c}}\end{array}$ & $\begin{array}{l}869.63^{\mathrm{d}} \\
869.56^{\mathrm{e}}\end{array}$ \\
\hline $1 s^{-1} 7 p^{3} P$ & 96.9756 & & 869.6147 & & \\
\hline${ }^{1} P$ & 96.9755 & & 869.6174 & $869.79^{c}$ & $869.73^{\mathrm{e}}$ \\
\hline $1 s^{-1} 8 p^{3} P$ & 96.9721 & & 869.7099 & & \\
\hline${ }^{1} P$ & 96.9720 & & 869.7127 & $869.87^{\mathrm{c}}$ & \\
\hline
\end{tabular}

${ }^{a}$ From LYP potential.

${ }^{\mathrm{b}} \mathrm{WF}[14]$.

${ }^{\mathrm{c}} \mathrm{CI}[21]$.

${ }^{\mathrm{d}}$ References [22,23].

${ }^{\mathrm{e}}$ References [21,24,25].

determinant is equal to the Hartree-Fock (HF) determinant. In the SLHF approach [3], we assume that the X-only KS determinant is also equal to the HF determinants for the excited states. Here the exchange potential is an exact nonvariational SLHF exchange potential. The KS energy eigenvalues obtained by solving the KS equation with this highquality exchange potential can serve as good zeroth-order excited-state energies. The accuracy and usefulness of the SLHF approach has been demonstrated by the recent successful calculations of singly, doubly, and triply Rydberg states of the valence electrons of He- and Li-like ions [3].

In this paper, we extend the SLHF density functional approach and GGA to the treatment of atomic inner-shell ex- cited states. Total and excitation energies of inner-shell excited states are calculated for closed-shell atomic systems: $\mathrm{Be}, \mathrm{B}^{+}, \mathrm{Ne}$, and $\mathrm{Mg}$. The results are in overall good agreement with available experimental and $a b$ initio theoretical data. We also present some new results for the highly excited inner-shell states.

In the SLHF density-functional approach, an electron spin-orbital is determined by the KS equation and the exchange potential is the SLHF exchange potential [3]. For an atomic system in the spherical coordinates, the spin-orbital $\varphi_{i \sigma}(\mathbf{r})$ of the $i$ th electron with spin $\sigma$ can be expressed as $\varphi_{i \sigma}(\mathbf{r})=R_{n l \sigma}(r) Y_{l m}(\theta, \phi) / r$, where, $n, l$, and $m$ are the principal quantum number, orbital angular momentum quantum number, and azimuthal quantum number of the electron, respectively, $Y_{l m}(\theta, \phi)$ is the spherical harmonic, and $R_{n l \sigma}(r)$ is the radial spin-orbital. The radial spin-orbital is governed by the radial $\mathrm{KS}$ equation [3]

$$
\left[-\frac{1}{2} \frac{d^{2}}{d r^{2}}+\frac{l(l+1)}{2 r^{2}}+v_{\mathrm{eff}}(r)\right] R_{n l \sigma}=\varepsilon_{n l \sigma} R_{n l \sigma},
$$

where, $\varepsilon_{n l \sigma}$ is the spin-orbital energy, $v_{\text {eff }}(r)=v_{\text {ext }}(r)+v_{H}(r)$ $+v_{x \sigma}(r)+v_{c \sigma}(r)$ is the radial effective potential, and $v_{\text {ext }}(r)$, $v_{H}(r), v_{x \sigma}(r)$, and $v_{c \sigma}(r)$ are the radial external potential, radial Hartree potential, radial SLHF exchange potential, and radial correlation potential, respectively. The radial SLHF exchange potential is given by [3]

$$
v_{x \sigma}(r)=-\frac{1}{4 \pi \rho_{\sigma}(r)} \sum_{\nu \nu^{\prime}}^{\nu_{\sigma} \nu_{\sigma}^{\prime}}\left[s_{\nu \nu^{\prime}}^{\sigma}(r)-c_{\nu \nu^{\prime}}^{\sigma}(r)\right],
$$

where $\nu=\{n l m\}, \nu_{\sigma}$ stands for a set of quantum numbers of the occupied spin-orbitals with the spin $\sigma, s_{\nu \nu^{\prime}}^{\sigma}(r)$ and $c_{\nu \nu^{\prime}}^{\sigma}(r)$ are the matrix elements given in [3], and $\rho_{\sigma}(r)$ is the spherically averaged spin-dependent electron density given by

TABLE IV. Total energies $(E)$ and excitation energies $(\Delta E)$ of inner-shell excited states $2 p^{-1} n s^{1,3} P(n=4 \sim 8)$ of $\mathrm{Mg}$. The groundstate energy -200.0744 (a.u.).

\begin{tabular}{rccccc}
\hline \hline & $\begin{array}{c}-E \text { (a.u.) } \\
\text { Present } \\
\text { work }^{\mathrm{a}}\end{array}$ & $\begin{array}{c}\Delta E(\mathrm{eV}) \\
\text { Present } \\
\text { work }^{\mathrm{a}}\end{array}$ & $\begin{array}{c}\text { Other } \\
\text { theory }^{\mathrm{b}}\end{array}$ & $\begin{array}{c}\text { Expt. } \\
\mathrm{MZ}^{\mathrm{c}}\end{array}$ & NE $^{\mathrm{d}}$ \\
\hline $2 p^{-1} 4 s^{3} P$ & 198.0557 & 54.9323 & 53.72 & 54.801 & 54.801 \\
${ }^{1} P$ & 198.0538 & 54.9849 & & 55.065 & 55.065 \\
$2 p^{-1} 5 s^{3} P$ & 198.0044 & 56.3272 & 55.99 & 56.278 & 56.280 \\
${ }^{1} P$ & 198.0038 & 56.3457 & & 56.544 & 56.545 \\
$2 p^{-1} 6 s{ }^{3} P$ & 197.9852 & 56.8499 & 56.56 & 56.777 & 56.785 \\
$2{ }^{1} P$ & 197.9849 & 56.8586 & & 57.039 & \\
$2 p^{-1} 7{ }^{3} P$ & 197.9759 & 57.1038 & & & \\
${ }^{1} P$ & 197.9757 & 57.1087 & & 57.302 & 57.305 \\
$2 p^{-1} 8 s{ }^{3} P$ & 197.9707 & 57.2464 & & & \\
${ }^{1} P$ & 197.9706 & 57.2478 & & & 57.456 \\
\hline
\end{tabular}

${ }^{\mathrm{a}}$ From LYP potential.

${ }^{\mathrm{b}} \mathrm{CIIOO}[26]$.

${ }^{\mathrm{c}}$ Reference [27].

${ }^{\mathrm{d}}$ Reference [28]. 
$\rho_{\sigma}(r)=(1 / 4 \pi) \sum_{n l}^{v_{\sigma}} w_{n l \sigma}\left(R_{n l \sigma} / r\right)^{2}$. The potential in Eq. (2) qualifies for excited-state calculation because it is selfinteraction free, has correct long-range behavior, and depends on symmetry of atomic state $[3,4]$. The exchange potential in Krieger, Li, and Iafrate (KLI) procedure [5], an approximation of the exact exchange optimized effective potential (OEP) [6], is an approximation of the SLHF exchange potential $[3,4]$. For atoms with nuclear charge $Z \geqslant 8$, the GGA correlation potential $v_{c \sigma}(r)$ is taken to be that proposed by Lee, Yang, and Parr (LYP) [7]. For atoms with $Z<8$, due to the overestimation of the LYP potential [3,7], the GGA correlation potential will be taken to be that proposed by Perdew and Wang (PW) [8].

The spin-orbitals are obtained by solving the radial KS equation (1) using the generalized pseudospectral (GPS) method [9]. The GPS method associated with an appropriate mapping technique can overcome difficulties due to singularity at $r=0$ and long-tail at large $r$ of Coulomb interaction. Furthermore, the GPS method allows for nonuniform and optimal spatial discretization with the use of only a modest number of grid points. It has been shown to be a very effective and efficient numerical algorithm for high-precision solution of KS equation [10-12]. From the spin-orbitals of an electronic configuration, a single Slater determinant for a

TABLE V. Total energies $(E)$ and excitation energies $(\Delta E)$ of inner-shell excited states $2 s^{-1} n p^{1,3} P(n=3 \sim 8)$ and $2 s^{-1} n s^{1,3} S$ $(n=4 \sim 8)$ of $\mathrm{Mg}$.

\begin{tabular}{|c|c|c|c|}
\hline States & $\begin{array}{c}-E \text { (a.u.) } \\
\text { Present } \\
\text { work }^{\mathrm{a}}\end{array}$ & $\begin{array}{c}\Delta E(\mathrm{eV}) \\
\text { Present } \\
\text { work }^{\mathrm{a}}\end{array}$ & $\begin{array}{l}\text { Other } \\
\text { theory }\end{array}$ \\
\hline $2 s^{-1} 3 p^{3} P$ & 196.6721 & 92.5820 & 91.72 \\
\hline${ }^{1} P$ & 196.6630 & 92.8288 & \\
\hline $2 s^{-1} 4 p^{3} P$ & 196.5181 & 96.7718 & 96.18 \\
\hline${ }^{1} P$ & 196.5170 & 96.8026 & \\
\hline $2 s^{-1} 5 p^{3} P$ & 196.4871 & 97.6164 & 97.11 \\
\hline${ }^{1} P$ & 196.4865 & 97.6317 & \\
\hline $2 s^{-1} 6 p^{3} P$ & 196.4738 & 97.9775 & \\
\hline${ }^{1} P$ & 196.4735 & 97.9863 & \\
\hline $2 s^{-1} 7 p^{3} P$ & 196.4669 & 98.1667 & \\
\hline${ }^{1} P$ & 196.4667 & 98.1721 & \\
\hline $2 s^{-1} 8 p^{3} P$ & 196.4628 & 98.2782 & \\
\hline${ }^{1} P$ & 196.4626 & 98.2820 & \\
\hline $2 s^{-1} 4 s^{3} S$ & 196.5499 & 95.9065 & 94.65 \\
\hline${ }^{1} S$ & 196.5447 & 96.0502 & \\
\hline $2 s^{-1} 5 s^{3} S$ & 196.4984 & 97.3079 & 97.07 \\
\hline${ }^{1} S$ & 196.4966 & 97.3585 & \\
\hline $2 s^{-1} 6 s^{3} s$ & 196.4792 & 97.8325 & 97.64 \\
\hline${ }^{1} S$ & 196.4783 & 97.8565 & \\
\hline $2 s^{-1} 7 s^{3} s$ & 196.4698 & 98.0872 & \\
\hline${ }^{1} S$ & 196.4693 & 98.1003 & \\
\hline $2 s^{-1} 8 s^{3} s$ & 196.4645 & 98.2301 & \\
\hline & 196.4642 & 98.2382 & \\
\hline
\end{tabular}

${ }^{\mathrm{a}}$ From LYP potential.

${ }^{\mathrm{b}} \mathrm{CIIOO}[26]$.
TABLE VI. Total energies $(E)$ and excitation energies $(\Delta E)$ of inner-shell excited states $1 s^{-1} n p^{1,3} P(n=3 \sim 8)$ and $1 s^{-1} n s^{1,3} S$ $(n=4 \sim 8)$ of $\mathrm{Mg}$.

\begin{tabular}{|c|c|c|c|}
\hline States & $\begin{array}{c}-E \text { (a.u.) } \\
\text { Present } \\
\text { work }^{\mathrm{a}}\end{array}$ & $\begin{array}{c}\Delta E(\mathrm{eV}) \\
\text { Present } \\
\text { work }^{\mathrm{a}}\end{array}$ & $\begin{array}{l}\text { Other } \\
\text { theory }^{b}\end{array}$ \\
\hline $1 s^{-1} 3 p^{3} P$ & 152.1433 & 1304.2822 & 1303.25 \\
\hline${ }^{1} P$ & 152.1353 & 1304.5002 & \\
\hline $1 s^{-1} 4 p^{3} P$ & 151.9851 & 1308.5868 & 1307.86 \\
\hline${ }^{1} P$ & 151.9839 & 1308.6192 & \\
\hline $1 s^{-1} 5 p^{3} P$ & 151.9536 & 1309.4453 & 1308.80 \\
\hline${ }^{1} P$ & 151.9533 & 1309.4521 & \\
\hline $1 s^{-1} 6 p^{3} P$ & 151.9401 & 1309.8108 & \\
\hline${ }^{1} P$ & 151.9399 & 1309.8179 & \\
\hline $1 s^{-1} 7 p^{3} P$ & 151.9331 & 1310.0018 & \\
\hline${ }^{1} P$ & 151.9329 & 1310.0062 & \\
\hline $1 s^{-1} 8 p^{3} P$ & 151.9290 & 1310.1145 & \\
\hline${ }^{1} P$ & 151.9289 & 1310.1172 & \\
\hline $1 s^{-1} 4 s^{3} s$ & 152.0171 & 1307.7160 & 1306.29 \\
\hline${ }^{1} S$ & 152.0144 & 1307.7895 & \\
\hline $1 s^{-1} 5 s^{3} S$ & 151.9649 & 1309.1362 & 1308.77 \\
\hline${ }^{1} S$ & 151.9640 & 1309.1618 & \\
\hline $1 s^{-1} 6 s^{3} S$ & 151.9454 & 1309.6660 & 1309.35 \\
\hline${ }^{1} S$ & 151.9450 & 1309.6780 & \\
\hline $1 s^{-1} 7 s^{3} S$ & 151.9360 & 1309.9226 & \\
\hline${ }^{1} S$ & 151.9358 & 1309.9297 & \\
\hline $1 s^{-1} 8 s^{3} S$ & 151.9307 & 1310.0663 & \\
\hline${ }^{1} S$ & 151.9306 & 1310.0706 & \\
\hline
\end{tabular}

${ }^{\mathrm{a}}$ From LYP potential.

${ }^{\mathrm{b}} \mathrm{CIIOO}[26]$.

multiplet state is constructed and its total energy calculated. For a multiplet state that cannot be determined by a single determinant, Slater's diagonal sum rule [13] is used to calculate the multiplet energy [3].

The above procedure is now used to calculate the total and excitation energies of inner-shell excited states of closed-shell atomic systems: $\mathrm{Be}, \mathrm{B}^{+}, \mathrm{Ne}$, and $\mathrm{Mg}$. For simplicity, an abbreviation $(n l)^{-1}\left(n^{\prime} l^{\prime}\right)$ is used to represent an electronic configuration of an electron in an inner subshell $(n l)$ being excited to a subshell $\left(n^{\prime} l^{\prime}\right)$. For instance, $1 s^{-1} 2 p$ of $\mathrm{Be}$ represents $1 s 2 s^{2} 2 p$. Throughout the paper, 1 a.u. $=27.2116 \mathrm{eV}$ is used.

In Table I we present the total and excitation energies of inner-shell excited states $1 s^{-1} n p^{1,3} P(n=2 \sim 8)$ of Be. For comparison, we also list the theoretical results of density work functional approach (WF) [14] and saddle-point complex-rotation method (SPCR) $[15,16]$, and available experimental results (Expt.) [17-19]. The maximum relative discrepancies of our total energies to the WF and SPCR results are $0.10 \%$ and $0.27 \%$, respectively. The maximum relative deviations of our excitation energies to the WF, SPCR, and experimental results are $0.18 \%, 0.35 \%$, and $0.37 \%$, respectively. Hence our results agree very well with the experimental and other theoretical results. 
To explore the feasibility of the approach to inner-shell excitation of atomic ions, we also apply the procedure to the calculation of inner-shell excitation of $\mathrm{B}^{+}$. The results for the excited states $1 s^{-1} n p^{1,3} P(n=2 \sim 8)$ are given in Table II along with the theoretical results of Dirac-Fock method (DF) [20] and experimental results [20]. The relative deviations of our excitation energies to the DF and experimental results are less than $0.32 \%$ and $0.29 \%$, respectively. Thus our results are in very good agreement with the experimental and other theoretical results.

In Table III we present the total and excitation energies of inner-shell excited states $1 s^{-1} n s^{1,3} S$ and $1 s^{-1} n p^{1,3} P(n=3$ $\sim 8$ ) of Ne. For comparison we also list the theoretical results of density work functional approach (WF) [14] and configuration-interaction model (CI) [21], and experimental results (Expt.) [22-25]. For the excited states $1 s^{-1} n s^{1,3} S$, the relative deviations of our total energies to the WF results are not more than $0.02 \%$. The relative discrepancies of our excitation energies to the experimental results are less than $0.02 \%$, while the maximum relative discrepancies of the WF and CI results to the experimental results are $0.08 \%$ and $0.03 \%$, respectively. For the excited states $1 s^{-1} n p{ }^{1,3} P$, the relative deviations of our total energies to the WF results are not more than $0.02 \%$. The maximum relative discrepancy of our excitation energies to the experimental results is $0.02 \%$ while the maximum relative deviations of the WF and CI results to the experimental results are $0.07 \%$ and $0.02 \%$, respectively. Thus our excitation energies are very close to the CI results and better than the WF data.

The total and excitation energies of inner-shell excited states $2 p^{-1} n s^{1,3} P, 2 s^{-1} n p^{1,3} P$ and $2 s^{-1} n s^{1,3} S$, as well as $1 s^{-1} n p^{1,3} P$ and $1 s^{-1} n s^{1,3} S$ for $n \leqslant 8$ of $\mathrm{Mg}$ are shown in Tables IV-VI, respectively. For comparison, the theoretical results of configuration-interaction calculation with improved and optimized orbitals (CIIOO) [26] and experimental results (Expt.) $[27,28]$ are also shown in these tables. For the excited states $2 p^{-1} n s{ }^{1,3} P$ in Table IV, the only ones for which experimental excitation energies are available, the CIIOO excitation energies are smaller than both the experimental results and our results. The maximum relative deviations of our excitation energies and the CIIOO results to the experimental results are $0.36 \%$ and $1.97 \%$, respectively. Thus our results are more accurate than the CIIOO results. For the excited states in Tables V and VI, the CIIOO excitation energies are again smaller than ours. The maximum relative deviations of our results to the CIIOO results are $0.94 \%, 1.33 \%, 0.08 \%$, and $0.11 \%$ for the excited states $2 s^{-1} n p^{1,3} P, 2 s^{-1} n s^{1,3} S, 1 s^{-1} n p^{1,3} P$, and $1 s^{-1} n s^{1,3} S$, respectively.

In summary, a SLHF density-functional approach is presented and applied to calculate the total energies and excitation energies of inner-shell excited states of closed-shell atomic systems: $\mathrm{Be}, \mathrm{B}^{+}, \mathrm{Ne}$, and $\mathrm{Mg}$. The results are in overall good agreement with the available, more sophisticated $a b$ initio theoretical results and experimental data. The relative discrepancies of our calculated excitation energies to the available experimental results are not more than $0.40 \%$, demonstrating that the SLHF density-functional approach provides a powerful and computationally efficient scheme for the accurate calculation of inner-shell excited states of closed-shell atomic systems within DFT. Finally, we present also a number of new results for highly excited inner-shell states.

This work is partially supported by the Chemical Sciences, Geosciences, and Biosciences Division of the Office of Basic Energy Sciences, Office of Science, U. S. Department of Energy, and by the National Science Foundation.
[1] M. P. Hertlein, et al., Phys. Rev. A 71, 022702 (2005).

[2] S. H. Southworth, et al., Radiat. Phys. Chem. 70, 655 (2004).

[3] Z. Zhou and S.-I. Chu, Phys. Rev. A 71, 022513 (2005).

[4] F. D. Sala and A. Görling, J. Chem. Phys. 115, 5718 (2001).

[5] J. B. Krieger, Y. Li, and G. J. Iafrate, Phys. Rev. A 46, 5453 (1992).

[6] J. D. Talman and W. F. Shadwick, Phys. Rev. A 14, 36 (1976).

[7] C. Lee, W. Yang, and R. G. Parr, Phys. Rev. B 37, 785 (1988).

[8] J. P. Perdew and Y. Wang, Phys. Rev. B 45, 13244 (1992).

[9] J. Wang, S. -I. Chu, and C. Laughlin, Phys. Rev. A 50, 3208 (1994).

[10] X. M. Tong and S.-I. Chu, Phys. Rev. A 55, 3406 (1997).

[11] X. Chu and S.-I. Chu, Phys. Rev. A 63, 023411 (2001).

[12] S.-I. Chu, J. Chem. Phys. 123, 062207 (2005).

[13] J. C. Slater, Quantum Theory of Atomic Structure Vol. II (McGraw-Hill, New York, 1960).

[14] A. K. Roy and B. M. Deb, Phys. Lett. A 234, 465 (1997).

[15] H. Lin, C. -S. Hsue, and K. T. Chung, Phys. Rev. A 64,
012709 (2001).

[16] H. Lin, C. -S. Hsue, and K. T. Chung, Phys. Rev. A 65, 032706 (2002).

[17] J. Jiménez-Mier, et al., J. Phys. B 32, 4301 (1999).

[18] C. D. Caldwell, et al., Phys. Rev. A 41, 542 (1990).

[19] E. Jannitti, et al., Opt. Commun. 63, 37 (1987).

[20] W. G. Lynam, et al., J. Phys. B 25, 3963 (1992).

[21] C. D. Schröter, et al., J. Phys. B 32, 171 (1999).

[22] A. P. Hitchcock and C. E. Brion, J. Phys. B 13, 3269 (1980).

[23] R. N. S. Sodhi and C. E. Brion, J. Electron Spectrosc. Relat. Phenom. 34, 363 (1984).

[24] M. Coreno, et al., Phys. Rev. A 59, 2494 (1999).

[25] L. Avaldi, et al., Phys. Rev. A 51, 5025 (1995).

[26] L. M. M. de Albuquerque Martins and C. E. Bielschowsky, Phys. Rev. A 56, 2720 (1997).

[27] W. C. Martin and R. Zalubas, J. Phys. Chem. Ref. Data 9, 1 (1980).

[28] G. H. Newsom, Astrophys. J. 166, 243 (1971). 